\title{
High Sensitivity Actinic Detection of Native Defects on EUVL Mask Blanks
}

\author{
Moonsuk Yi, \\ Center for X-Ray Optics, Lawrence Berkeley National Laboratory, \\ Berkeley, California 94720 \\ Tsuneyuki Haga, \\ NTT Telecommunications Energy Laboratories, \\ Atsugi, Kanagawa 243-0198 Japan \\ Chris Walton, \\ Lawrence Livermore National Laboratory, Livermore, CA 94550 \\ Jeffrey Bokor \\ Center for X-Ray Optics, Lawrence Berkeley National Laboratory, \\ Berkeley, California 94720 \\ and EECS Department, University of California, Berkeley, California 94720
}

\begin{abstract}
We present recent experimental results of actinic extreme ultraviolet lithography mask blank defect inspection. The detection sensitivity of the current actinic inspection system is predicted to be able to reach approximately $30 \mathrm{~nm}$ in cross correlation experiments done with commercial visible-light, SEM and AFM inspection tools. Random, native defects identified using the visible-light tool were scanned by the actinic tool and the scattering characteristics were compared. Scanning electron microscopy and atomic force microscopy (AFM) characterization of selected defects were also performed for physical measurement. We found a defect whose size was as small as $60 \mathrm{~nm}$ and AFM analysis showed that this defect was a $3 \mathrm{~nm}$ high substrate defect that was smoothed-out by the multilayer coating.
\end{abstract}

Electronic mail: msyi@lbl.gov 


\section{INTRODUCTION}

Extreme Ultraviolet Lithography (EUVL) is one of the leading next-generation lithography candidates for fabricating integrated circuits with feature size of $70 \mathrm{~nm}$ or below EUVL utilizes a reflective mask with patterned absorbers on a multilayer reflection-coated mask blank. Detecting and characterizing defects on the multilayer mask blank is a very important technical issue because the requirement for the size and number of printable defects is stringent for advanced masks. While several inspection strategies are being actively pursued for detecting defects on EUVL mask blanks, an actinic (at-wavelength) inspection capability is essential for learning the nature of defects at the developmental stage of the technology and for eventually qualifying more practical non-EUV inspection methods.

In EUVL masks, critical defects can occur either in the absorber pattern, the multilayer reflection coating, or due to contamination of the finished mask. Defects can affect the amplitude and phase of the wavefront reflected from the mask and can significantly reduce the process window for printing features 2 . For example, when a defect disrupts the multilayer growth, it can induce a significant drop in local reflectivity (opaque defect or amplitude defect). On the other hand, if the multilayer is deposited conformally over a particle residing on the mask blank substrate, the resultant conformal topography induces a localized phase error in the reflected electric field (phase defect). For the $100 \mathrm{~nm}$ device generation, the size of the smallest "printable" $\pi$-phase defect on a $4 \times$ reduction mask has been calculated ${ }^{3}$ to be $52 \mathrm{~nm}$. Under some conditions, the multilayer growth process can smooth-out the effects of small substrate particle defects" ${ }^{4}$. A method of "defect compensation" for some defects in the multilayer blank has been proposed, but has not yet been demonstrated ${ }^{3}$. 
Visible wavelength laser-based optical scattering wafer inspection tools have been used for routine inspection of EUVL mask blanks. Although those commercial optical inspection tools have a high throughput of close to 100 wafers per hour with high sensitivity, it is uncertain whether visible inspection tools can find all of the EUV-printable defects. This is because of the significant difference in optical properties of the multilayer coating between the visible and the EUV wavelength. Therefore, at least at the initial developmental stage of multilayer deposition and inspection technology, an actinic (at-wavelength) inspection might be required to detect all the defects on an EUVL mask blank. Actinic inspection directly probes the effect of a defect on the reflected electric field and helps to assess the printability of the defect. An actinic inspection system also helps to establish a non-actinic inspection strategy for ultimate commercial use via cross correlation of the EUV response of defects with their visible response.

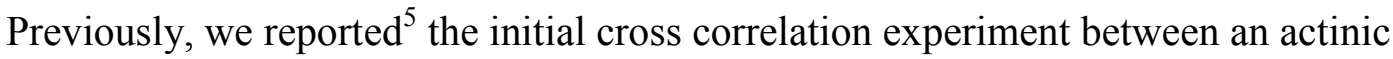
EUVL defect scanner and an optical scattering inspection tool in which randomly occurring, native defects found in one tool can be studied with each of the others. Recently our actinic EUVL defect scanner was greatly improved with increased detection sensitivity and scanning speed. Previously, we spent 80 hours to scan $1 \mathrm{~cm}^{2}$ with $80 \mathrm{~nm}$ sensitivity, but now we need 30 hours to scan $1 \mathrm{~cm}^{2}$ with $30 \mathrm{~nm}$ sensitivity. The $30 \mathrm{~nm}$ sensitivity can be predictable based on the signal-to-noise ratio of our tool and the cross correlation results with PSL-calibrated optical inspection tools. In this paper, we report new cross correlation experimental results after the system improvements, including detailed characterization of some interesting defects. We also show the results of a defect counting experiment over $6.25 \mathrm{~cm}^{2}$ area, especially the characteristics of small defects that were not detected by optical inspection tools. 


\section{THE CURRENT SENSITIVITY OF THE ACTINIC INSPECTION TOOL}

The actinic EUV defect scanner, which is based on a raster scanning EUV microscope, has been discussed in previous publications ${ }^{5}$. Briefly, when the focused EUV beam is incident on a defect, the defect induces a decrease in the intensity of the specularly reflected beam (bright field detection) and scattering of photons into nonspecular directions (dark field detection). The basic schematics of the actinic inspection system are shown in Figure 1(a). The detector assembly is constructed for simultaneous bright field and dark field detection. For dark field detection, a microchannel plate detector is used with a hole at the center to pass the specularly reflected beam. The small focal spot on the sample is formed by imaging an EUV-illuminated aperture using a pair of curved, glancing angle mirrors in the Kirkpatrick-Baez (KB) configuration. The smallest spot size we have produced so far is $2.5 \mu \mathrm{m}$ by $4 \mu \mathrm{m}$.

Defects much smaller than the focal spot size can be detected as small variations of the bright-field and/or dark-field signals. The detection sensitivity depends on the signal-to-noise ratio of the bright and dark field signals at a fixed time constant. In this system, beam intensity variation after passing through the small entrance aperture is the biggest contributor to the noise in bright and dark field signals. The incident beam motion relative to the fixed aperture position caused by beamline mirror heating, vibration, and synchrotron source motion causes the intensity noise. To suppress this noise, we developed a feedback loop to cause the aperture to track the brightest portion of the beam spot. This not only suppresses slow intensity drift, it also minimizes the level of acoustic intensity noise for a given amount of vibration because the aperture sits at a local intensity maximum. Additionally, we used notch filter electronics to knock out certain specific vibration frequencies. Finally, digital signal post-processing further suppresses noise. We also increased the scanning rate of the system without any sacrifice of 
signal-to-noise ratio by carefully optimizing the data acquisition system. The detection sensitivity of the current actinic inspection tool is now estimated to be around $30 \mathrm{~nm}$.

\section{CROSS CORRELATION EXPERIMENT}

In what we refer to as the cross correlation experiment, defects on an EUVL mask blank are first found by a visible inspection tool along with their coordinates and sizes based on the optical scattering cross section. Later the same sample is loaded in the EUV inspection tool and a small area around each defect is scanned to probe the EUV response of the defects. Once a defect is found, it can be further analyzed with atomic force microscopy and scanning electron microscopy with compositional analysis capability, because studying the morphology and composition of defects is very important for revealing the source of the defects and possibly controlling them.

In Ref. 6, Jeong, et al. reported successful cross correlation experiments between the optical inspection tool (KLA Tencor SP1) and our actinic inspection tool. In this section, we report new cross correlation results with enhanced detection capability and also report detailed characterizations of some interesting defects.

For the cross correlation experiment, a multilayer-coated 8 inch silicon wafer was first inspected by commercial optical inspection tools, such as SP1 from KLA-Tencor and Constellation from ADE. Then, the actinic inspection tool inspected the wafer with accurate coordinate registration between the optical and actinic tools. The correlation among signals from the optical inspection tool and the bright/dark field signals of the actinic tool is shown in Figure 1. Figure 1(b) shows the correlation between the bright field signal and the estimated defect size from the SP1 for 23 defects and 1(c) shows the correlation between the bright field and dark field actinic signals. The bright field signal is defined as the fractional reduction of the bright field 
detector output relative to the neighboring clear region. The dark field signal is defined as the fractional increase in the dark field detector output relative to the background level. The majority of defects, which were classified as "on-trend" defects, have a good correlation between the bright field signal and SP1 signal with several exceptions. These "off-trend" defects also showed off-trend characteristics in the correlation between the bright field and dark field actinic signals shown in Figure 1(c).

Special attention goes to the off-trend defects, since most on-trend defects are opaque defects: these are most likely particles lying on top of the multilayer blank and these defects can be eliminated in a sufficiently clean environment. The off-trend defects were inspected by AFM and SEM to determine their actual shape, size, morphology and composition. Among them, three defects denoted by small circles in Figure 1(c) will be described in detail on the following paragraphs.

The \#1 defect in Figure 1 is classified as off-trend because of the strong dark field signal compared to the bright field signal. Figure 2 shows the bright and dark field images and the AFM image of this defect. It induced a $16.9 \%$ decrease in the specularly reflected intensity, and $653 \%$ increase in the non-specularly scattered intensity with respect to the background scattered intensity from the multilayer blank. The estimated sizes from the bright and dark field signals were $640 \mathrm{~nm}$ and $1.2 \mu \mathrm{m}$. This size estimation is derived from the cross correlation between the calibrated optical inspection tool and actinic signals for the on-trend defects. As can be seen in the AFM image, this defect was a clustered defect consisting of a big defect and some surrounding small debris. Usually a defect induces more scattering at its perimeter as compared to its interior. Therefore this clustered defect, having relatively large perimeter to area ratio shows enhanced scattering. In contrast to the \#1 defect, the \#2 defect in Figure 1 is classified off- 
trend because of the strong bright field signal compared to the dark field signal. Figure 3 shows the bright and dark field images and an SEM image. As this defect is significantly larger than our beam spot size $(2.5 \mu \mathrm{m}$ by $4 \mu \mathrm{m})$, we can directly determine the size from the actinic scan images. In the bright field image in Figure 3, the size is approximately $12 \mu \mathrm{m}$, which is consistent with the SEM image, but the dark field image of the defect is much smaller than the bright field image. This defect induced a $19.5 \%$ decrease in the bright field intensity, and a $26 \%$ increase in the dark field intensity with respect to the clear area. The estimated size from the dark field signal level was only $85 \mathrm{~nm}$. We postulate that the $26 \%$ dark field scattering signal arises from scattering by the small particles within the $12 \mu \mathrm{m}$ circle seen in the SEM image, and the scattering signal level from the big, circular body of this defect was in the same range as the blank region. Energy dispersive spectroscopy (EDS) data showed this defect to have an organic component, and the AFM scan showed this defect as a 40-nm-high bump with $12 \mu \mathrm{m}$ diameter. Further analysis is in progress now to determine the precise nature of this defect.

The \#3 defect is shown in detail in Figure 4. It is also quite large; the size was $28 \mu \mathrm{m}$. We observed an unusual wavelength response at this defect; the non-specular scattering characteristics were different at different parts of this defect. The plots in Figure 4 show the wavelength response of the scattered intensity at two different locations within the defect. The peak wavelength was shifted to a shorter wavelength at the upper left part of the defect, where we observed in the SEM image that the particle was rolled up. This shape has changed the scattering angle and therefore the wavelength dependence. The EDS data shows that this defect consists of Si and Mo, thus we suspect that this defect is a flake of multilayer coating, perhaps from inside the coating chamber. 


\section{DEFECT COUNTING EXPERIMENT}

Another question crucial to establishing the correlation between the non-EUV inspection tool and the EUV inspection tool is whether all the defects found by the EUV inspection tool can be found by visible inspection tools. This experiment, complementary to the cross correlation experiment, involves scanning a clean area of EUVL mask blank, which is previously proven to be defect-free by optical inspection tools, looking for defects with the actinic inspection system. This type of experiment is referred to as the "at-wavelength defect counting experiment ${ }^{\prime \prime}$.

We report the results of a new defect counting experiment from a total of $6.25 \mathrm{~cm}^{2}$ area over 3 mask blanks. Relatively clean cross correlation samples were scanned in certain areas that the optical inspection had determined to be defect-free. The total EUV scanning time was 30 hours per $\mathrm{cm}^{2}$ with time constant of $4 \mathrm{~ms}$ and the pixel size was $1 \mu \mathrm{m}$ by $4 \mu \mathrm{m}$ with dwell time of $4 \mathrm{~ms}$ per pixel. A scan was divided into smaller regions of size $0.5 \mathrm{~cm}^{2}$ so that the individual scan file size is manageable. The beamline alignment was automatically re-optimized every hour to reduce the beam position drift. After each $0.5 \mathrm{~cm}^{2}$ scan, the data were processed to pick up any defect above the threshold. If there was a signal above the threshold, a small region ( $50 \mu \mathrm{m}$ by $50 \mu \mathrm{m}$ typically) was scanned with time constant of $100 \mathrm{~ms}$ in order to determine whether the signal is a false count or a real defect. This procedure was repeated until the full $6.25 \mathrm{~cm}^{2}$ over 3 samples was covered.

During actinic defect counting of a $6.25 \mathrm{~cm}^{2}$ area, 28 defects were found. These defects were re-inspected by visible inspection tools to identify particles added by the actinic tool as well as wafer handling. After eliminating the "adders", at least, three "actinic-only" defects were 
identified. Figure 5 shows one of these actinic-only defects. Its size as seen from the AFM inspection was about $60 \mathrm{~nm}$ and the height was less than $3 \mathrm{~nm}$. This defect appears to be a phase defect smoothed out by the multilayer coating. Most of the actinic-only defects were smaller than $100 \mathrm{~nm}$, which is just below the detection capability of the current visible inspection tools. Further characterization of these small defects is in progress.

\section{SUMMARY}

We have improved the detection capability of our actinic EUVL mask blank inspection tool down to $30 \mathrm{~nm}$ defect size, and the scanning time for $1 \mathrm{~cm}^{2}$ with $30 \mathrm{~nm}$ sensitivity is now 30 hours. Formerly it was 80 hours with $80 \mathrm{~nm}$ sensitivity. A cross correlation experiment between non-actinic inspection tools and the actinic inspection tool shows good correlation for the majority of defects. The characteristics of some off-trend defects have been investigated. We detected a small native phase defect, whose size is about $60 \mathrm{~nm}$, through an actinic defect counting experiment, and this defect was below the detection capability of the current visible EUVL mask blank inspection tools. Actinic inspection has been demonstrated as a highly sensitive inspection technique for providing effective feedback to commercial inspection tool development and to help understand the nature of native defects.

\section{ACKNOWLEDGMENTS}

This work is supported by the Extreme Ultraviolet Lithography Limited Liability Company (EUV LLC), the Office of Energy Research, Basic Energy Sciences, of the U.S. Department of Energy under contract No. DE-AC03-76SF00098, SRC contract No. 96-LC-460 and DARPA 
No. grant MDA972-97-1-0010. Authors would like to thank Cindy Larson of Livermore laboratory for her efforts on AFM and SEM inspections.

\section{REFERENCES}

${ }^{1}$ C. W. Gwyn, R. Stulen, D. Sweeney, and D. Attwood, J. Vac. Sci. Technol. B 16, 3142 (1998).

${ }^{2}$ K. Nguyen, Ph.D. dissertation, University of California, Berkeley, CA, 1994.

${ }^{3}$ A. K. Ray-Chaudhuri, G. Cardinale, A. Fisher, P.-Y. Yan, and D. W. Sweeney, J. Vac. Sci. Technol. B 17, 3024 (1999).

${ }^{4}$ G. F. Cardinale, A. K. Ray-Chaudhuri, A. Fisher, P. S. J. Mangat, J. Wasson, P. B. Mirkarimi, and E. Gullikson, J. Vac. Sci. Technol. B 18, 2944 (2000).

${ }^{5}$ S. Jeong, L. Johnson, S. Rekawa, C. C. Walton, S. T. Prisbrey, E. Tejnil, J. H.Underwood, and J. Bokor, J. Vac. Sci. Technol. B 17, 3009 (1999).

${ }^{6}$ S. Jeong, C. Lai, S. Rekawa, C. C. Walton, and J. Bokor, Proc. SPIE 3997, 431 (2000).

${ }^{7}$ M. Yi, S. Jeong, S. Rekawa, and J. Bokor, J. Vac. Sci. Technol. B 18, 2930 (2000). 
FIG. 1. (a) Schematic diagram of our actinic defect inspection system. (b) Relationship between bright field (BF) signal and defect size estimated from the signal strength of optical inspection tools. (c) Relationship between bright field (BF, specular reflection) signal and dark field (DF, non-specular reflection, scattering) signal of 32 defects that the actinic tool found through the cross correlation experiment. The off-trend defects are identified in both plots by triangles.

FIG. 2. Defect \#1 in Fig.1. Strong dark field scattering signal is observed compared to the bright field signal. (a) Bright field image: $16.9 \%$ decrease in reflected light intensity, (b) Dark field image: $653 \%$ increase in scattered light intensity, (c) AFM image showing clustered defects, (d) Far-field scattering pattern ${ }^{7}$ at the dark field detector plane captured by CCD a camera. FIG. 3. Defect \#2 in Figure 1. Weak dark field scattering signal is observed compared to the bright field signal. (a) Bright field image: $19.5 \%$ decrease in reflected light intensity, (b) Dark field image: $26 \%$ increase in scattered light intensity, (c) SEM micrograph showing some debris and the actual size of $12 \mu \mathrm{m}$.

FIG. 4. Defect \#3 in Figure 1. Big flake defect. (a) Bright field image: $94.2 \%$ decrease in reflected light intensity, (b) Dark field image: $278 \%$ increase in scattered light intensity, (c) SEM micrograph showing the actual size of $28 \mu \mathrm{m}$, (d) Wavelength scanning at two different positions showing unusual peak-wavelength shift at upper left (UL) corner.

FIG. 5. Images of a small phase defect found in a defect counting experiment; (a) Dark field image from actinic inspection, (b) AFM image of the same defect. The estimated size from the actinic inspection was less than $70 \mathrm{~nm}$. The AFM scan indicates a defect approximately $60 \mathrm{~nm}$ wide and less than $3 \mathrm{~nm}$ high. 

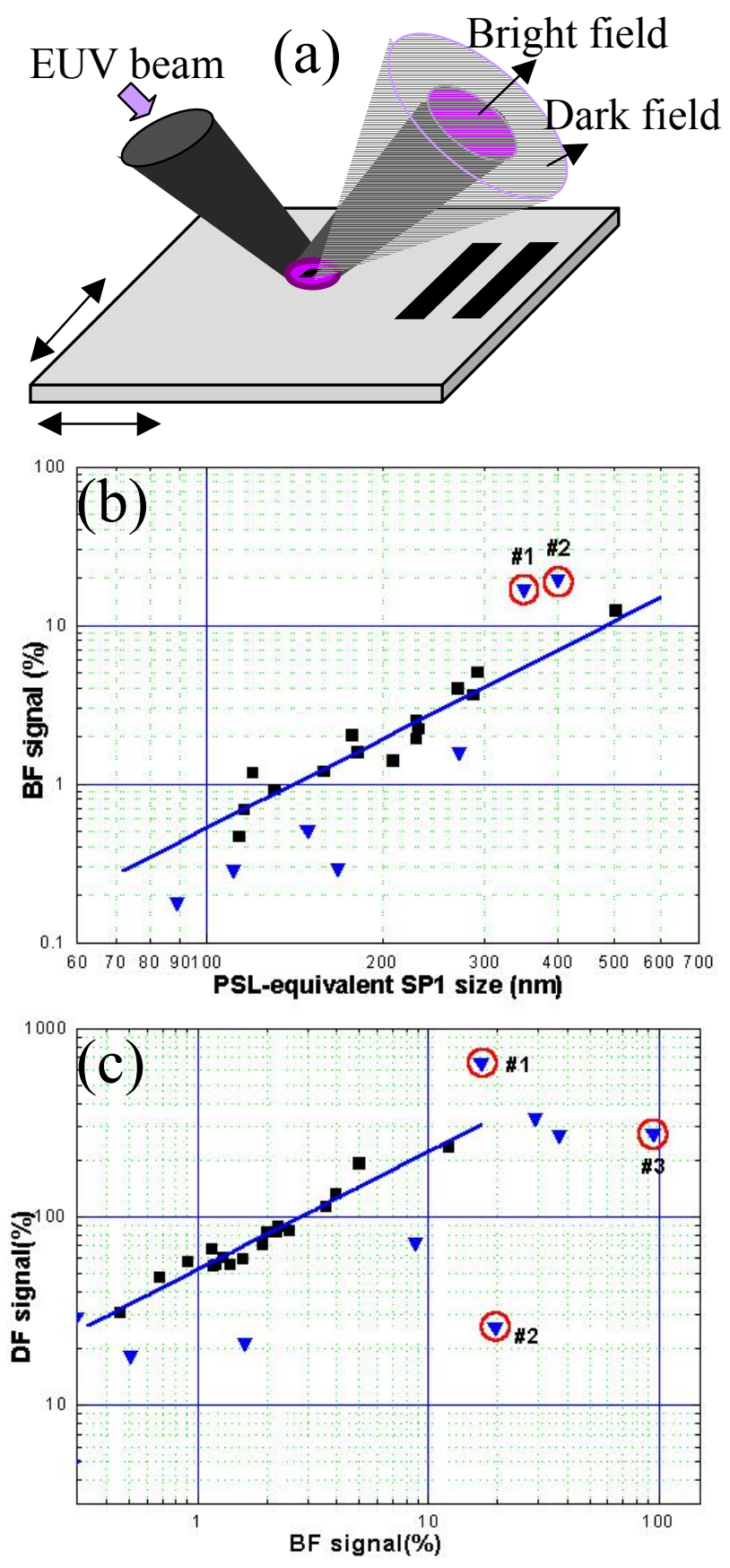

Fig 1 


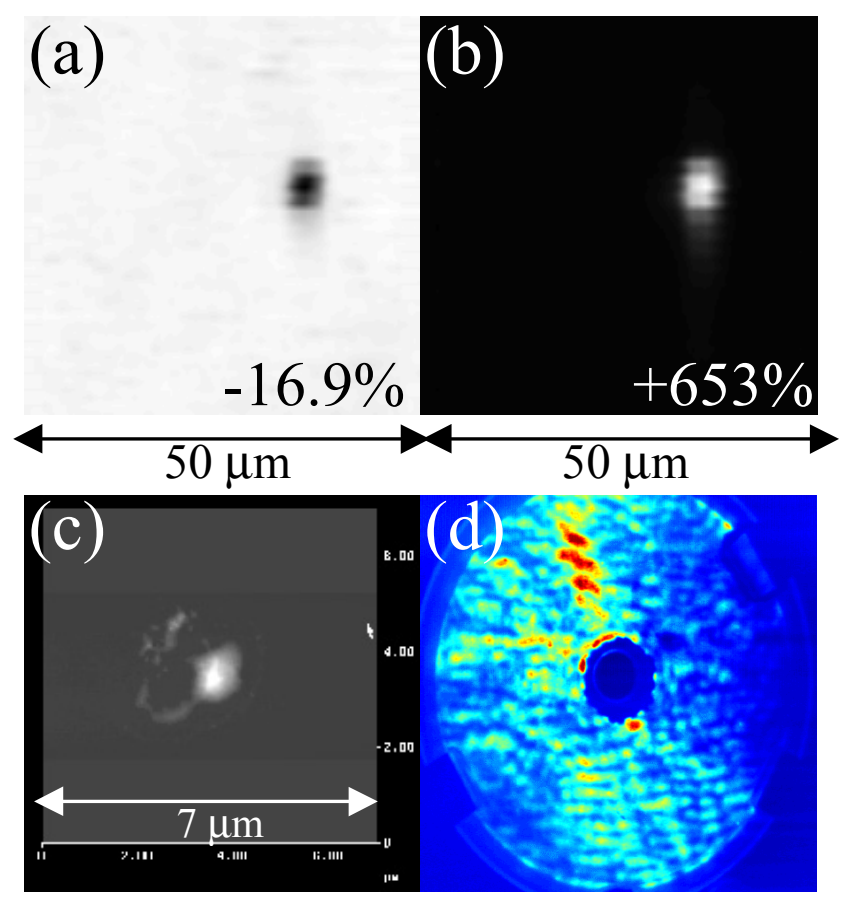

Fig 2 


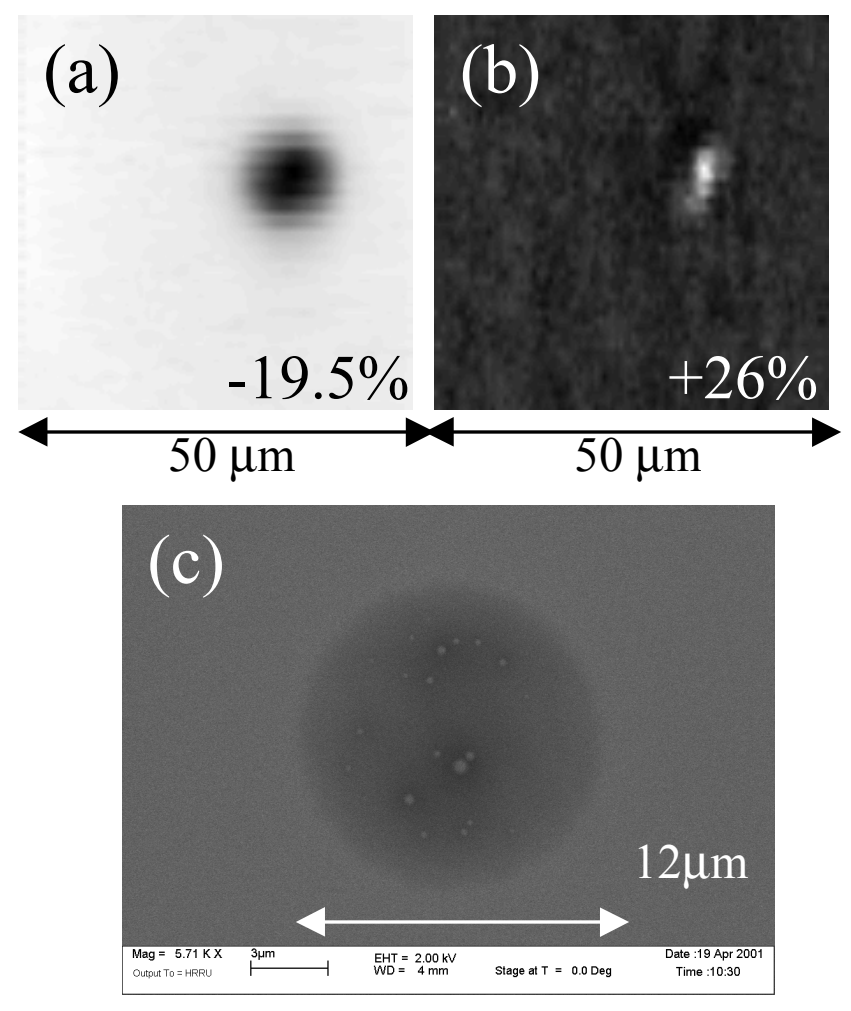

Fig 3 

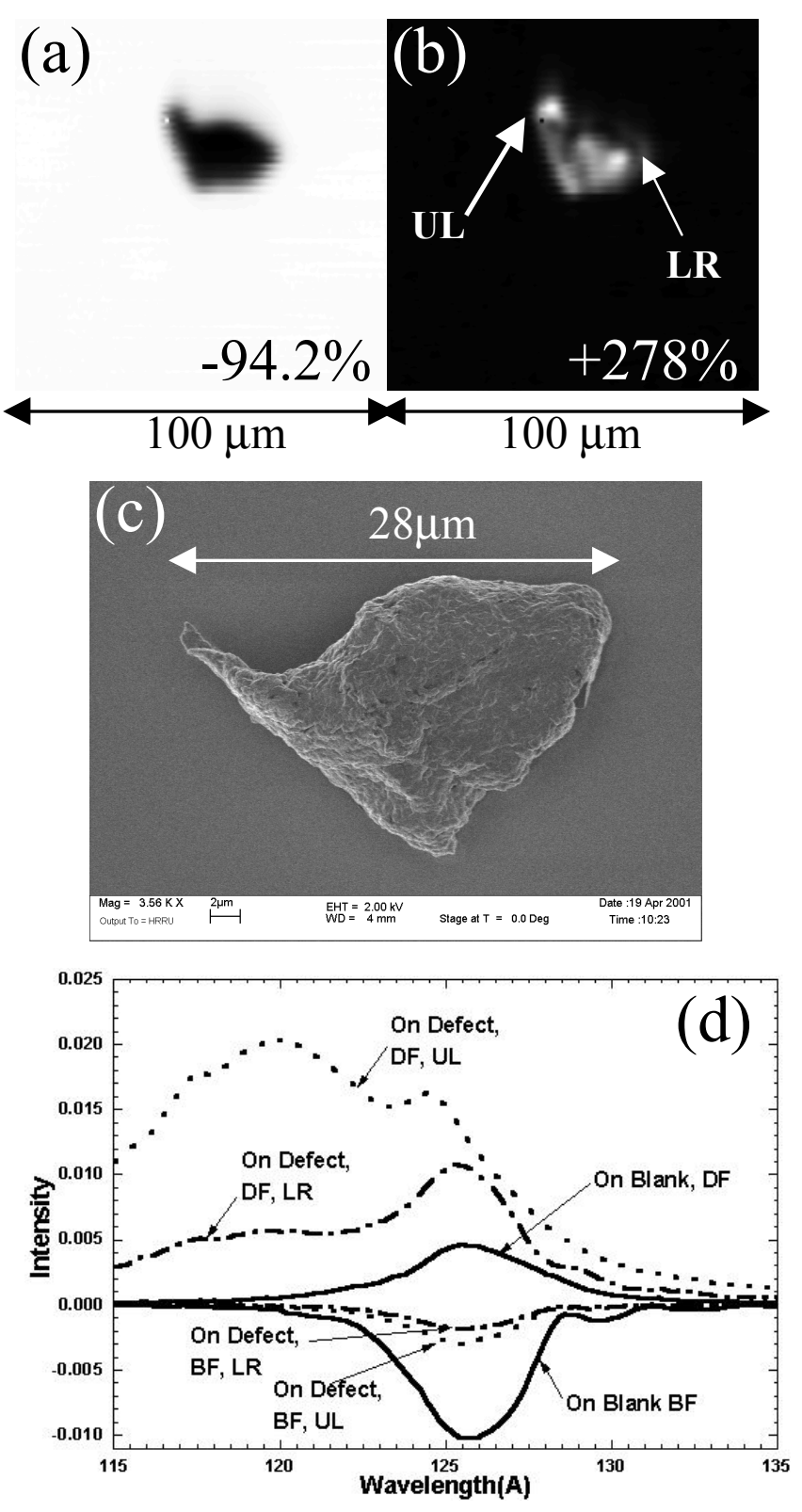

Fig 4 


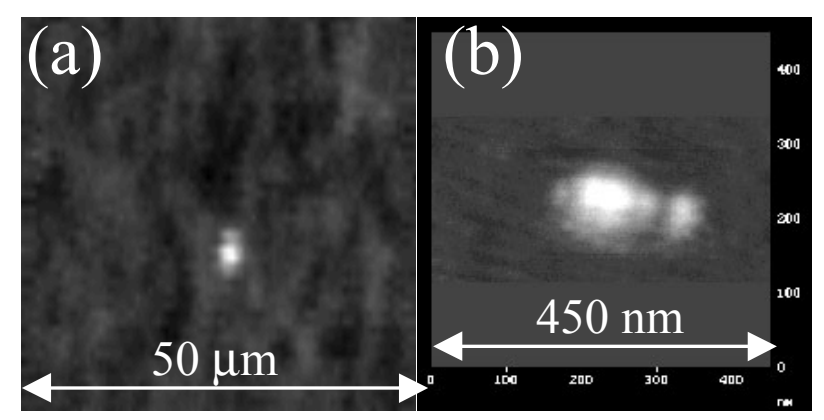

Fig 5 